\title{
L-carnitine treatment of insulin resistance: A systematic review and meta-analysis
}

\author{
Ying $\mathrm{Xu}^{1, B, D}$, Wenjie Jiang ${ }^{2, C, D}$, Guochang Chen ${ }^{1, E}$, Wenjiao Zhu ${ }^{3, B}$, Weiliang Ding ${ }^{3, E}$, \\ Zhijun Ge $e^{4, A}$, Yongfei Tan ${ }^{5, A}$, Tieliang Ma ${ }^{3}, \mathrm{E}, \mathrm{F}$, Guoxing Cui ${ }^{1, A, F}$ \\ ${ }^{1}$ Department of Gastroenterology, the Affiliated Yixing Hospital of Jiangsu University, Yixing, Jiangsu, China \\ ${ }^{2}$ Department of Anesthesiology, the Affiliated Yixing Hospital of Jiangsu University, Yixing, Jiangsu, China \\ ${ }^{3}$ Central Laboratory, the Affiliated Yixing Hospital of Jiangsu University, Yixing, Jiangsu, China \\ ${ }^{4}$ Department of Critical Care Medicine, the Affiliated Yixing Hospital of Jiangsu University, Yixing, Jiangsu, China \\ ${ }^{5}$ Department of Cardiac \& Thoracic Surgery, the Affiliated Yixing Hospital of Jiangsu University, Yixing, Jiangsu, China \\ A - research concept and design; $\mathrm{B}$ - collection and/or assembly of data; $\mathrm{C}$ - data analysis and interpretation; \\ $D$ - writing the article; $E$ - critical revision of the article; $F$ - final approval of article
}

\section{Address for correspondence}

Guoxing Cui

E-mail: staff1762@yxph.com

\section{Funding sources}

This work was supported by Fund of Science and Technology of Yixing (2013-20), Fund of Clinical Science and Technology of Wuxi (ML201304) and Fund of Six Best Talent of Jiangsu (WSN-024).

\section{Conflict of interest}

none declared

Received on May 18, 2015

Revised on June 11, 2015

Accepted on January 29, 2016

\begin{abstract}
Background. L-carnitine has been used for several years as an adjuvant therapy in oxidative stress, blood sugar, high-sensitivity C-reactive protein (CRP), anemia, etc. However, the efficacy of L-carnitine treating insulin resistance (IR) remains controversial. Homeostasis model assessment of Insulin Resistance (HOMA-IR) is widely used in the clinical evaluation of patients with IR.

Objectives. A meta-analysis, including randomized controlled trials (RCTs), was performed to assess the effect of $\mathrm{L}$-carnitine on HOMA-IR patients.

Material and methods. The Cochrane Library, PubMed, and EMBASE databases were systematically searched to identify RCTs which evaluated the effects of L-carnitine on HOMA-IR patients. We screened relevant studies according to predefined inclusion and exclusion criteria. In the selected articles, we extracted the data: study design, sample size, age, L-carnitine dose and regimen, body mass index (BMI) of patients, mode of administration, study duration and study outcomes.

Results. A total of 5 studies were included for the meta-analysis. The result showed $L$-carnitine was useful in the treatment of IR (WMD $-0.724, \mathrm{Cl}-0.959-0.488, \mathrm{p}<0.0001$ ). Evaluation at $3,6,9,12$ months, the $\mathrm{p}$-values were $0.875,0.165,0.031,0,007$, respectively.

Conclusions. L-carnitine was useful in treating patients with IR. L-carnitine can treat IR more effectively with prolonging the medication time. However, more RCTs with long-term L-carnitine treatment of IR are needed to confirm the viewpoint.
\end{abstract}

Key words: insulin resistance, meta-analysis, L-carnitine

DOI

10.17219/acem/61609

Copyright

Copyright by Author(s)

This is an article distributed under the terms of the

Creative Commons Attribution Non-Commercial License

(http://creativecommons.org/licenses/by-nc-nd/4.0/) 
Many factors can cause insulin resistance (IR), such as infection, acidosis, excessive stress, smoking, exposure to secondhand smoking. ${ }^{1}$ IR means the cells require higher concentrations of insulin in order to produce insulin response. ${ }^{2}$ Plasma IR, induced by high insulin and high sugar content, can lead to metabolic syndrome, gout and type II diabetes. ${ }^{3-5}$

Homeostasis model assessment of Insulin Resistance (HOMA-IR) was first proposed by Turner of the University of Oxford research group in 1985 and has been widely used in the clinical evaluation of insulin sensitivity. ${ }^{6}$ HOMA-IR was calculated as [serum insulin (IU/L) $\times$ $\times$ Plasma glucose $(\mathrm{mmol} / \mathrm{L})] / 22.5{ }^{6}$ The HOMA model is used to estimate cell function from fasting plasma insulin and glucose concentrations. A higher score of HOMA-IR indicates more severe IR. HOMA-IR of the normal is less than 2.68 .

L-carnitine is an important transporter of long-chain fatty acids which is primary synthesized in the liver and kidney. ${ }^{7}$ Its shortage will impair the use of fat as fuel, which will result in an acute metabolic decompensation. ${ }^{8}$ Previously, L-carnitine was mainly used to lose weight and to assist in dialyzing kidneys. ${ }^{9,10}$ Recently, several studies have reported its use in treating IR. ${ }^{11,12}$ However, the real effect of L-carnitine on IR remains unclear. Therefore, we performed this meta-analysis.

\section{Data sources and search strategy}

Two investigators (XY and JWJ) independently searched the Cochrane Central Register of Controlled Trials, PubMed, and EMBASE databases from inception to 2014-2010. The search terms were "L-carnitine" and "insulin resistance". If the 2 investigators had disagreements, it would be resolved by a $3^{\text {rd }}$ person (MTL).

\section{Study selection}

When selecting studies, we obeyed the following criteria. Inclusion criteria: Randomized Controlled Trials (RCTs) which evaluated the effects of L-carnitine on HOMA-IR. HOMA-IR as the index in assessing the degree of IR in patients. Treatment time was less than 6 months. Publications written in English.

Exclusion criteria: Non-randomized controlled trials. Results were not evaluated by HOMA-IR. Studies did not offer the detailed data.

\section{Study quality assessment}

The Newcastle-Ottawa Scale was used to assess the methodological quality of the included studies based on their selection of participants, comparability of groups and exposure/outcome ascertainment. A total score of 6 or higher (max score, 9) was used to identify higher-qual- ity studies. The quality of these studies was evaluated by 2 authors (XY and JWJ) independently. If 2 investigators had disagreements, it would be resolved by the $3^{\text {rd }}$ person (MTL).

\section{Data extraction and management}

The following data was extracted: study design, sample size, mean age of patients, body mass index (BMI) of patients, L-carnitine dose and regimen, mode of administration, study duration and study outcomes. The data was independently extracted by 2 authors (XY and JWJ) and disagreements were resolved by the $3^{\text {rd }}$ author (MTL).

\section{Data synthesis and statistical analysis}

Using the data derived from these RCTs, we used the weighted mean differences (WMDs) to report the continuous outcomes, and 95\% confidence intervals (CIs) were also calculated. ${ }^{13}$ Subgroup analysis was used to assess the differences at evaluation time of 3, 6, 9, 12 months, respectively. $\mathrm{I}^{2}$ was used to assess the heterogeneity among the selected studies. The meta-analyses were performed with STATA 12.0 software (copyright 1985-2011 StataCorp LP).

\section{Results}

\section{Study selection and characteristic}

Searching the database with time limit from the database set up date to October 2014. A total of 581 studies were included in the search and then 314 studies based on animal experiments, theme inconsistent, letters, review and meta-analysis were excluded. After reading the full text content roughly, we excluded the papers which were not randomized controlled trial, not relevant to Lcarnitine and not relevant to IR. Then, we further analyzed the rest of the data. We further excluded 22 articles which presented with different indicator of IR and units of IR. Finally, 5 studies were included in the meta-analysis. ${ }^{11,12,14-16}$ Details of the study selection process and reasons for study exclusion were presented in Fig. 1.

These 5 studies involved a total of 631 patients, in which 319 were treated with L-carnitine and 312 with placebo. Study duration ranged from 4 weeks to 12 months. The characteristics of included studies were summarized in Table 1 . In the 5 articles, the Nos score (95\% CI) was $6 \pm 2$. The detail rules of NOS scoring system are listed in Table 2. 

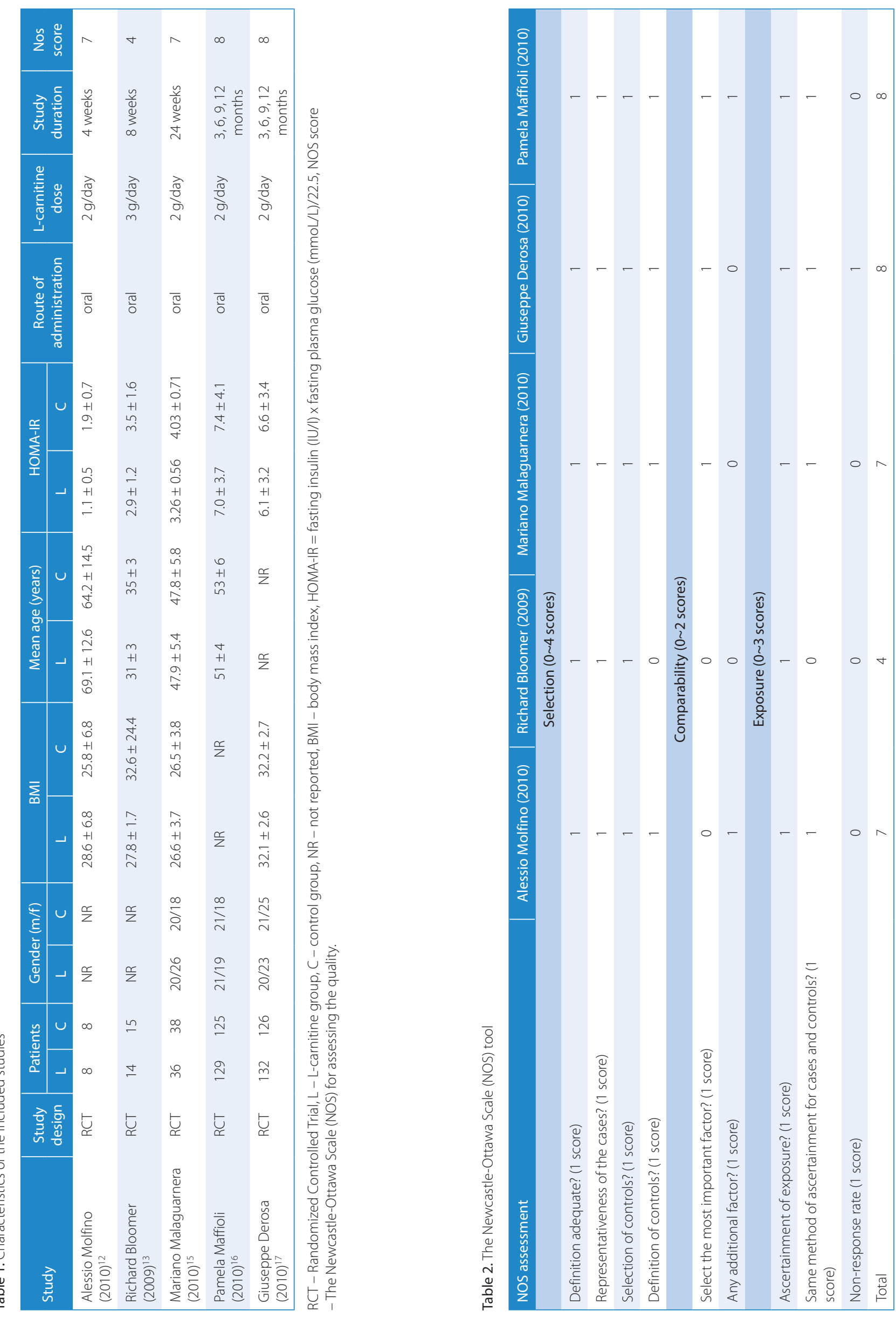


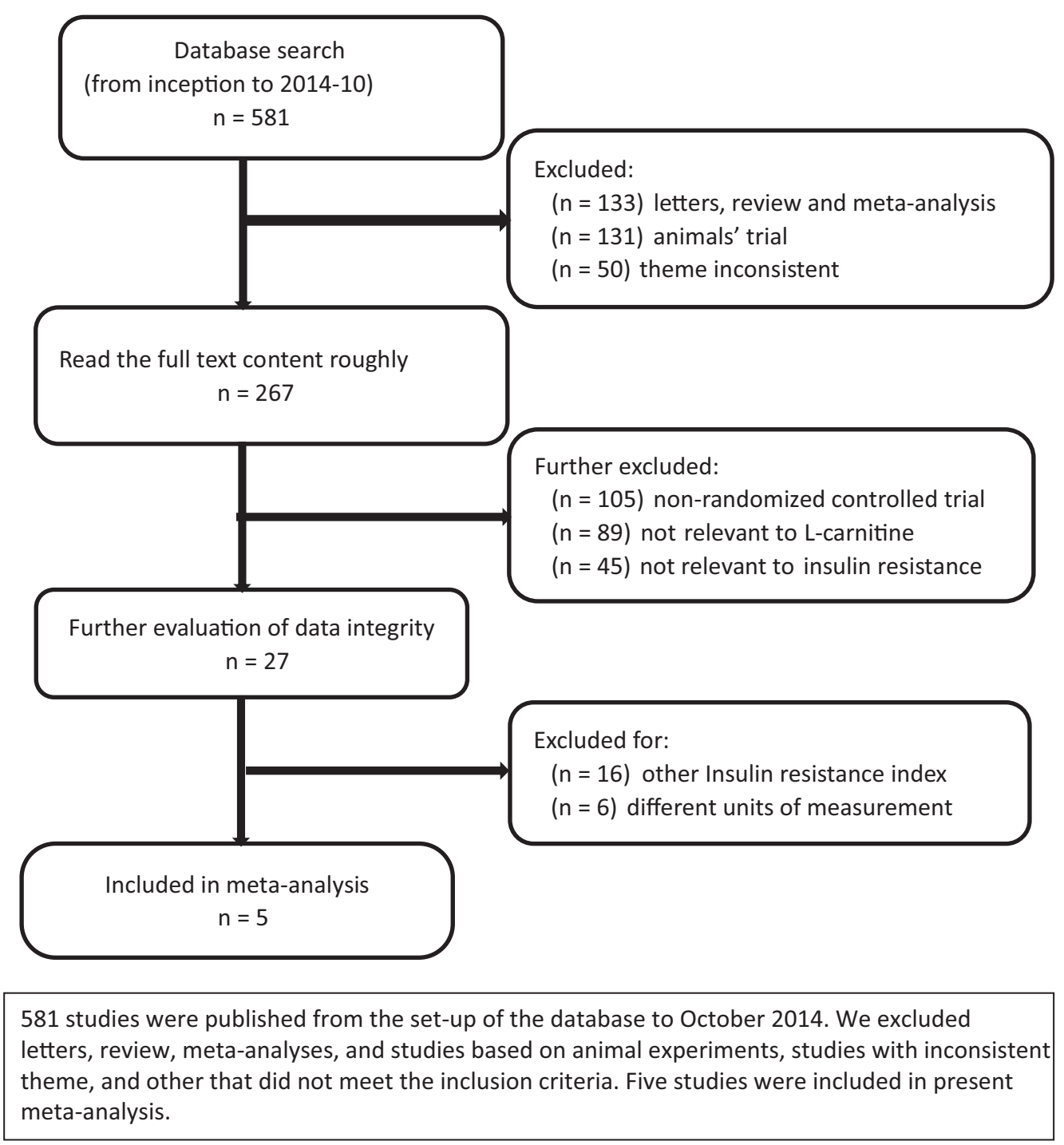

Fig. 1. Flow diagram of literature searching and selecting

\section{Effects of L-carnitine treatment on HOMA-IR}

The results showed that L-carnitine could treat HOMAIR compared to the placebos (WMD -0.724, CI -0.959 TO $-0.488, \mathrm{p}<0.0001)$ Fig. 2. Little heterogeneity existed in the analysis $\left(\mathrm{I}^{2}=0 \%\right)$. The results of comparisons among different evaluation time indicated that the treatment of L-carnitine became more and more effective with time elapsed (3, 6, 9, 12 months: $\mathrm{p}=0.875,0.165,0.031$, 0.007 , respectively) Table 3 .

\section{Discussion}

IR is a state in which a given concentration of insulin produces a less-than-expected biological effect. ${ }^{17}$ IR widely exists in a variety of diseases not just in diabetes. ${ }^{8,18}$ IR makes up a broad clinical spectrum including obesity, glucose intolerance, diabetes, and the metabolic syndrome which will impair the patients' quality of life,
Table 3. Different evaluation time comparison chart

\begin{tabular}{|l|c|c|c|}
\hline Treatment time & WMD $[95 \% \mathrm{Cl}]$ & $\mathrm{I}^{2}$ & p-value \\
\hline 3 months & $-0.062(-0.762$ to 0.638$)$ & $0.0 \%$ & 0.875 \\
6 months & $-0.459(-1.099$ to 0.181$)$ & $0.0 \%$ & 0.165 \\
9 months & $-0.201(-0.385$ to -0.018$)$ & $0.0 \%$ & 0.031 \\
12 months & $-0.257(-0.443$ to -0.071$)$ & $0.0 \%$ & 0.007 \\
\hline
\end{tabular}

More detailed comparative analysis was conducted, based on 2 of the 5 enrolled literatures, which had longer experimental time. The analysis results showed that the longer the treatment time, the smaller $p$-value, indicating that with the extension of treatment time, the effect of L-carnitine is more significant.

lower their survival rate and produce a heavy economic burden on society. ${ }^{9,15,19,20}$ However, no therapy has been found to treat IR effectively. Recently, several studies researched the use of L-carnitine in treating IR. ${ }^{21}$

L-carnitine is an important transporter of long-chain fatty acids that can be synthesized endogenously from lysine and methionine or obtained from the diet. ${ }^{16} \mathrm{~L}$-carnitine is widely used in the maintenance of hemodialysis to 


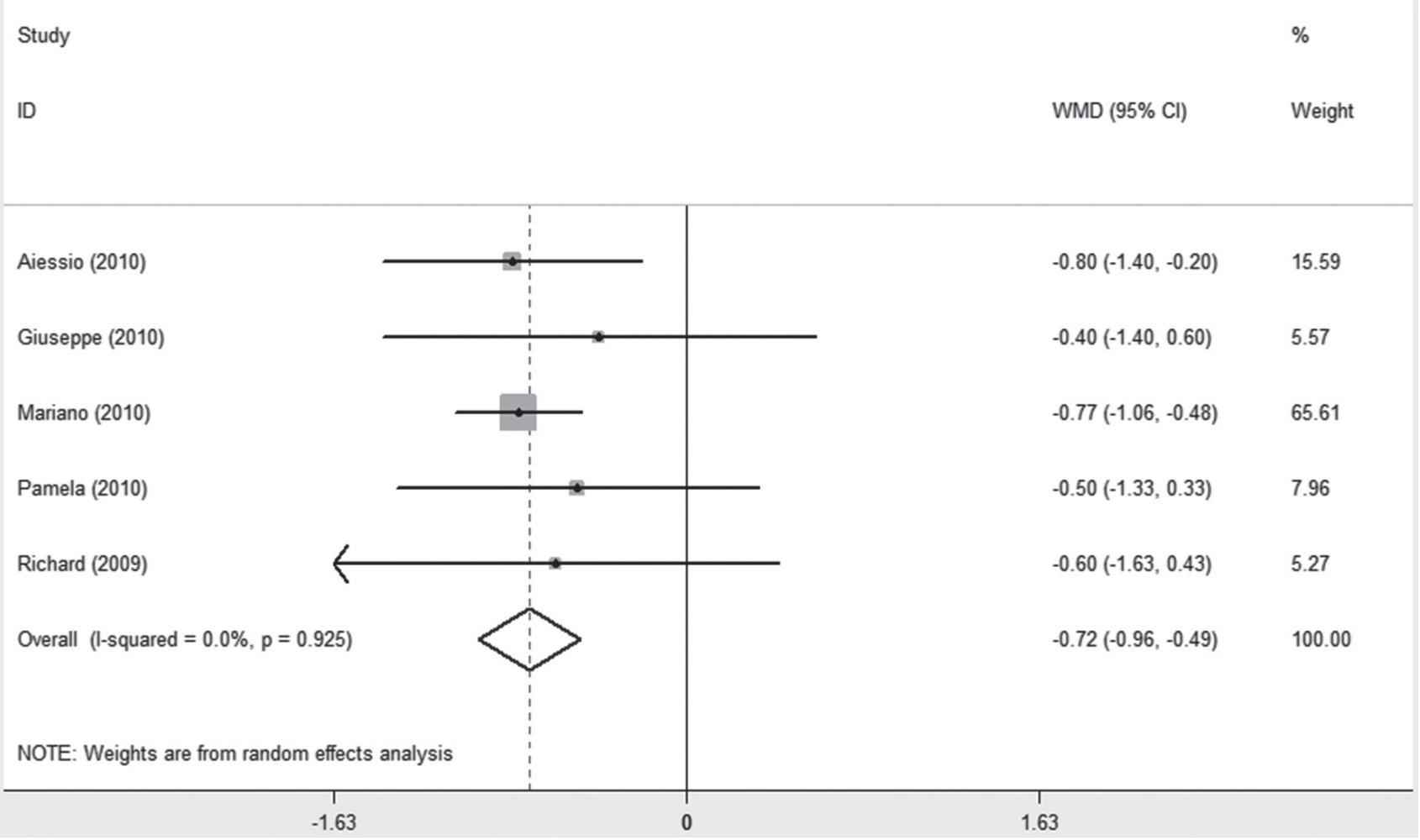

Fig. 2. Forest plot of meta-analysis of L-carnitine on the HOMA-IR in patients

replenish carnitine depletion and has several important proven physiological functions, such as offering nutrition, remedying anemia, adjusting dyslipidemia, curing hyperparathyroidism, developing cognitive functions and enhancing the quality of life in patients. ${ }^{12,22}$

HOMA-IR is a useful index in evaluating IR and is widely used in the evaluation of IR. Several studies showed that HOMA-IR and euglycemic hyperinsulinemic glucose clamps (the IR's gold standard). ${ }^{23}$

Therefore, we performed the meta-analysis to evaluate the effect of L-carnitine on HOMA-IR. Five papers of L-carnitine treating in IR were included in the metaanalysis. The results showed that L-carnitine was useful in reducing the HOMA-IR. Little heterogeneity existed in the analysis. Two of the selected articles included the evaluation time separately at 3, 6, 9, 12 months, then we made a further analysis. The results showed that the treatment of L-carnitine became more and more effective with time elapsed.

The meta-analysis had several advantages. Firstly, the heterogeneity among the included studies was insignificant. Secondly, all of the articles included in the metaanalysis were randomized controlled trials. Thirdly, the articles included in the meta-analysis have high quality by using NOS score tool which has high validity and reliability. Lastly, the paper assessed the efficacy of L-carnitine in 3, 6, 9, 12 months separately.

However, this meta-analysis had some limitations. Firstly, the use of mean \pm SD of HOMA-IR was proba- bly unsuitable as it is unlikely to be normally distributed and quartile range seemed to be more suitable. However, all the results in the included studies were expressed as mean $\pm \mathrm{SD}$, then we contacted authors to raw data but didn't get a response. Secondly, the experimental time was not long enough in the included studies, and the treatment period did not have uniform criteria. Thirdly, uncontrollable factors may exist in the included 2 groups, such as lifestyle, eating habits and the effects of these factors, which can impact the results significantly. Fourthly, the sample size was small, as only 631 patients were included. This may not represent all the patients well. Lastly, only 2 articles included the evaluation time of 3, 6, 9, and 12 months studies separately and the result may not be persuasive.

Taking all the above into consideration, we need more RCTs with longer treatment time and better control of the interference factors to further research the efficiency of L-carnitine, which could guide clinical practice and provide sound advice for patients more efficiently.

\section{Conclusion}

L-carnitine was valid in HOMA-IR. The longer is the treatment duration, the more significant is the treatment effect. However, more RCTs with an adequate sample size and hard endpoints are needed to define the effect of L-carnitine on patients. 


\section{References}

1. Shulman GI. Cellular mechanisms of insulin resistance. J Clin Invest. 2000;106:171-176.

2. Gutch M, Kumar S, Razi SM, Gupta KK, Gupta A. Assessment of insulin sensitivity/resistance. Indian J Endocrinol Metab. 2015;19:160-164.

3. Güçlü F, Özmen B, Hekimsoy Z, Kirmaz C. Effects of a statin group drug, pravastatin, on the insulin resistance in patients with metabolic syndrome. Biomed Pharmacother. 2004;58:614-618.

4. Shores NJ,Keeffe EB. Is oral I-acyl-carnitine an effective therapy for hepatic encephalopathy? Review of the literature. Digestive Diseases and Sciences. 2008;53:2330-2333.

5. Mingrone G. Carnitine in type 2 diabetes. Ann N Y Acad Sci. 2004; 1033:99-107.

6. Bonora E, Targher G, Alberiche M, et al. Homeostasis model assessment closely mirrors the glucose clamp technique in the assessment of insulin sensitivity: Studies in subjects with various degrees of glucose tolerance and insulin sensitivity. Diabetes Care. 2000;23, 57-63.

7. Mingorance $C$, Rodriguez-Rodriguez $R$, Justo $M L$, Alvarez de Sotomayor M, Herrera MD. Critical update for the clinical use of L-carnitine analogs in cardiometabolic disorders. Vasc Health Risk Manag. 2011, 7, 169-176.

8. Takenaka T, Kanno Y, Ohno Y, Suzuki H. Key role of insulin resistance in vascular injury among hemodialysis patients. Metabolism. 2007; 56:153-159.

9. Mihalik SJ, Goodpaster BH, Kelley DE, et al. Increased levels of plasma acylcarnitines in obesity and type 2 diabetes and identification of a marker of glucolipotoxicity. Obesity (Silver Spring). 2010; 18:1695-1700.

10. Yang SK, Xiao L, Song PA, Xu X, Liu FY, Sun L. Effect of L-carnitine therapy on patients in maintenance hemodialysis: A systematic review and meta-analysis. J Nephrol. 2014;27:317-329.

11. Molfino A, Cascino A, Conte C, Ramaccini C, Rossi Fanelli F, Laviano $A$. Caloric restriction and $L$-carnitine administration improves insulin sensitivity in patients with impaired glucose metabolism. J Parenter Enteral Nutr. 2010;34:295-299.

12. Malaguarnera M, Gargante MP, Russo $C$, et al. L-carnitine supplementation to diet: a new tool in treatment of nonalcoholic steatohepatitis: A randomized and controlled clinical trial. Am J Gastroenterol. 2010;105:1338-1345.
13. Higgins JP and Thompson SG. Quantifying heterogeneity in a meta-analysis. Stat Med. 2002;21:1539-1558.

14. Bloomer RJ, Fisher-Wellman KH, Tucker PS. Effect of oral acetyl $\mathrm{L}$-carnitine arginate on resting and postprandial blood biomarkers in pre-diabetics. Nutr Metab (Lond). 2009;6:25.

15. Vaneckova I, Maletinska L, Behuliak M, Nagelova V, Zicha J, Kunes J. Obesity-related hypertension: Possible pathophysiological mechanisms. J Endocrinol. 2014;223:R63-78.

16. Derosa G, Maffioli P, At Salvadeo S, et al. Sibutramine and L-carnitine compared to sibutramine alone on insulin resistance in diabetic patients. Internal Medicine. 2010;49:1717-1725.

17. Schooneman MG, Vaz FM, Houten SM and Soeters MR. Acylcarnitines: Reflecting or inflicting insulin resistance? Diabetes. 2013;62:1-8.

18. Emami Naini A, Moradi M, Mortazavi M, et al. Effects of oral L-carnitine supplementation on lipid profile, anemia, and quality of life in chronic renal disease patients under hemodialysis: A Randomized, double-blinded, placebo-controlled trial. J Nutr Metab. 2012;2012:1-6.

19. Biolo G, Stulle M, Bianco F, et al. Insulin action on glucose and protein metabolism during L-carnitine supplementation in maintenance haemodialysis patients. Nephrol Dial Transplant. 2008;23:991-997.

20. Malaguarnera $M$, Vacante $M$, Bertino $G$, et al. The supplementation of acetyl-L-carnitine decreases fatigue and increases quality of life in patients with hepatitis $C$ treated with pegylated interferonalpha 2b plus ribavirin. J Interferon Cytokine Res. 2011;31:653-659.

21. Hong ES, Kim EK, Kang SM, et al. Effect of carnitine-orotate complex on glucose metabolism and fatty liver: A double-blind, placebo-controlled study. J Gastroenterol Hepatol. 2014;29:1449-1457.

22. Malaguarnera M, Vacante M, Giordano M, et al. Oral acetyl-L-carnitine therapy reduces fatigue in overt hepatic encephalopathy: A randomized, double-blind, placebo-controlled study. Am J Clin Nutr. 2011;93:799-808.

23. Donjacour CE, Aziz NA, Overeem S, Kalsbeek A, Pijl H, Lammers GJ. Glucose and fat metabolism in narcolepsy and the effect of sodium oxybate: A hyperinsulinemic-euglycemic clamp study. Sleep. 2014; 37:795-801. 